\title{
Der Gott des Da-zwischen
}

\section{Einleitung - worum es in diesem Beitrag geht}

„Dazwischen“: Es spricht einiges dafür, das Dazwischen als einen Aspekt der Wirklichkeit zu sehen, dem kein besonderes Interesse gilt. Einen Abstand und damit ein Dazwischen muss man zwar zur Kenntnis nehmen. Aber Abstände und ein Dazwischen überwindet man, wichtiger scheint auf den ersten Blick der Ausgangspunkt und der Endpunkt. Andererseits: „Der Weg ist das Ziel“ - so eine bekannt Werbung - also der Abstand und das Dazwischen sind interessant...

Was damit auf jeden Fall ins Blickfeld gerückt ist, das wird auch in der Philosophie zum Thema. Sie widmet sich, so sagt man nicht zu Unrecht, den letzten, den wesentlichen Fragen. Und das Wesentliche ist das bleibend Gültige. Philosophie ist interessiert am Wesen der Dinge, an dem was bleibend gültig ist. Übergänge und Veränderungen - die gibt es natürlich - wer wollte es leugnen? - aber als entscheidend erscheint das, was bleibt. In einer solchen Sicht geht das Interesse am Dazwischen jeglicher Art verloren.

Und damit sind wir bereits mitten in unserem Thema. ${ }^{1}$ Denn der Teilbereich der Philosophie, der sich mit den grundlegenden Kategorien der Wirklichkeit befasst, wird herkömmlicherweise als Ontologie bezeichnet. Man kann ihr

1 Der vorliegende Aufsatz wurde in italienischer Übersetzung publiziert in: B. Körner, Il Dio dell'in-mezzo, „Sophia: Ricerche su i fondamenti e la correlazione dei saperi“ 8 (2016) nº 2, S. 61-71. 
Thema mit der Frage charakterisieren: Was macht die Wirklichkeit zur Wirklichkeit? Kein Zweifel: damit zielt die Ontologie auf Grundlegendes.

\section{Unsere grund-legenden, oft unbedachten Überzeugungen. Oder: Was ist das - Ontologie?}

Als Menschen versuchen wir, uns in der Wirklichkeit zu orientieren. Die Zeitung lesen, zur Straßenbahnstation gehen, die Wäsche waschen, über ein Problem nachdenken, einen Brief schreiben - immer ist das verbunden mit einer bestimmten Idee davon, was die Wirklichkeit ausmacht und wie man damit umgehen kann bzw. muss. Dabei geht es nicht nur - um bei den eben genannten Beispielen zu bleiben - um das Wissen, was z.B. eine Zeitung ist oder eine Straßenbahn.

Sinnvolle, oft unbedachte Vor-Urteile

Es geht auch um Überzeugungen, die noch grundlegender sind und normalerweise als selbstverständlich angesehen werden - so z.B. die Überzeugung,

- dass die Wirklichkeit zu beschaffen ist, dass etwas nicht zugleich das eine und das Gegenteil davon sein kann;

- dass Dinge bleiben was sie sind, und dass man daher unter den gleichen Umständen damit auch in Zukunft so umgehen kann wie heute;

- dass verschiedene Gegenstände trotz markanter Unterschiede „ihrem Wesen nach" das Gleiche sind.

Über solche und andere Grundideen geben wir uns normalerweise keine Rechenschaft, wir denken darüber nicht nach, sondern gehen einfach davon aus, dass die Wirklichkeit tatsächlich so beschaffen ist, wie es in diesen Überzeugungen zum Ausdruck kommt. Und solche und ähnliche Überzeugungen helfen uns, mit der Wirklichkeit zu Recht zu kommen.

\section{Weitreichende Weltanschauungen}

Aber dann gibt es auch andere Grundideen. Sie sind umstritten und werden je nach Weltanschauung oder religiöser Einstellung unterschiedlich gesehen. - So gehen Menschen in der Regel davon aus, dass die Wirklichkeit, die sie wahrnehmen, unabhängig davon existiert, ob sie sie wahrnehmen oder nicht. Allerdings hat es auch - vermutlich wenige - andere gegeben, die das in Frage gestellt haben. Sie vertreten das Prinzip, dass eine vom Erkennen unabhängige Wirklichkeit nicht existiert: esse est percipi (so der anglikanische Bischof George Berkeley (+ 1753)). 
- Es gibt Menschen, die halten das Geistige für ein Produkt der Materie; andere gehen davon aus, dass es vor allem Materiellen das Geistige gegeben hat.

- Religiöse Menschen gehen in der Regel davon aus, dass es gewissermaßen „außerhalb“ der den Menschen zugänglichen Wirklichkeit auch eine „jenseitige“ Wirklichkeit gibt. Andere schließen das aus.

Das Gemeinsame solcher grundlegenden Überzeugungen über die Wirklichkeit besteht darin, dass sie wissenschaftlich nicht bewiesen werden können, sondern der wissenschaftlichen Arbeit immer schon vorausgehen. Das Kriterium für die Annahme solcher Überzeugung besteht darin, dass sie zwar nicht bewiesen werden können, dass man sie aber daran messen kann, ob bzw. wie sie sich im Umgang mit der Wirklichkeit bewähren.

\section{Ontologie - Ontologien}

Die philosophische Disziplin, die sich mit diesen grundlegenden Gegebenheiten der Wirklichkeit befasst, wird - so wurde bereits am Beginn dieses Beitrags gesagt - Ontologie genannt. Je nach Position kann sie unterschiedlich ausgeprägt sein - z.B. als religiöse, als atheistische, als materialistische oder als idealistische Ontologie usw.

Außerdem kann eine Ontologie unterschiedlich vorgehen. Sie kann (1.) beschreibend verfahren, d.h. erheben, welche ontologischen Annahmen einer bestimmten Darstellung der Wirklichkeit zugrunde liegen („deskriptive Ontologie“) oder sie kann (2.) eine bestimmte Ontologie systematisch entwerfen und vorgeben („normative Ontologie“). ${ }^{2}$ Klaus Hemmerle, um dessen Thesen $z u$ einer trinitarischen Ontologie im Folgenden gehen wird, hat beides getan. Einerseits hat er sich mit der Frage befasst, welche ontologischen Annahmen den mystischen Intuitionen der Gründerin der Fokolar-Bewegung zugrunde liegen. ${ }^{3}$ Mit seinen Thesen zu einer trinitarischen Ontologie ist er darüber hinausgegangen und hat auf systematische Weise eine Ontologie begründet und vorgestellt. Da er sich aber auch in seinen Thesen auf die Spiritualität der Einheit stützt, muss diese jetzt vorgestellt werden.

2 Diese Unterscheidung hat Peter F. Strawson vorgenommen; er spricht von einer „deskritpiven“ und einer „revisionären Metaphysik“ - vgl. P. F. Strawson, Einzelding und logisches Subjekt, Stuttgart 1972, S. 9.

3 So im posthum veröffentlichten Aufsatz K. Hemmerle, Lontologia del „Paradiso” “49, „Sophia: Ricerche su i fondamenti e la correlazione dei saperi“ 6 (2014) n 2, S. 127-137. 
2. Das „Charisma der Einheit“. Oder: Die Spiritualität, die Klaus Hemmerle zu denken gab

Wenn man die geistlichen Intuitionen von Chiara Lubich in den Blick nehmen will, muss man vor allem auf einige wenige Ereignisse in ihrem Leben zu sprechen kommen. In ihrer (geistlichen) Lebensgeschichte wird deutlich, wie sie durch einige markante geistliche Erfahrungen, vor allem im Jahr 1949, einen unverwechselbaren Zugang zum überlieferten christlich-katholischen Glauben gefunden hat.

Zwei Brennpunkte: die Einheit und der verlassene Jesus

An erster Stelle ist eine dramatische Episode im Luftschutzkeller während eines Bombenangriffs auf ihre Heimatstadt Trient zu nennen. ${ }^{4}$ Mit ihren Freundinnen schlägt sie das Evangelium auf und stößt auf die Abschiedsreden im Johannes-Evangelium: „Alle sollen eins sein, wie du, Vater, in mir bist und ich in dir bin, sollen auch sie in uns sein“ (Joh 13,34). Dieses „eins sein“ verstehen Chiara Lubich und ihre Freundinnen als das Testament Jesu. Es zur Lebensform zu machen erscheint ihnen als die richtige Antwort auf die Liebe Gottes.

Dazu kommt ein zweites Ereignis. Ein Gespräch mit einem Kapuziner-Pater. ${ }^{5}$ Er stellt der jungen Frau die Frage, wo ihrer Meinung nach Jesus am meisten gelitten hat. Die Antwort, die er selbst auf seine Frage gibt: Jesus habe am meisten gelitten in seiner Verlassenheit am Kreuz: „Mein Gott, mein Gott, warum hast Du mich verlassen“ (Mk 15,34). Auch diese Auskunft wird zu einem Angelpunkt der von Chiara Lubich entdeckten Spiritualität.

Die gegenseitige Liebe, die zur Einheit führt, als Ziel auf der einen Seite, und auf der anderen Seite der Weg zu diesem Ziel - der Weg bis in die Gottverlassenheit. Damit sind die Eckpfeiler der Fokolar-Spiritualität, der Spiritualität der Einheit genannt.

\section{Gott-Liebe}

Den Hintergrund dafür bildet ein Gottesbild, das seine charakteristische Ausprägung darin findet, dass in Jesus Gott Mensch geworden ist und - bis zum Tod am Kreuz. Darin zeigt sich eine Liebe, über die hinaus keine größere

4 Vgl. zu diesen biographischen Ausführungen in erster Linie das Interview mit Chiara Lubich, das unter dem Titel erschienen ist: Ch. Lubich, Die Welt wird eins. Franca Zambonini im Gespräch mit der Gründerin der Fokolar-Bewegung, München 1991, S. 50.

5 Vgl. Ch. Lubich, Die Welt wird eins, S. 49. 
gedacht werden kann. In der Mitte dieser Spiritualität geht es nicht um Gott an sich, gewissermaßen einen statischen Gott, sondern um Gott, der sich hingibt. Der „Gott der Chiara Lubich“ kann unmöglich als ein in sich ruhender Urgrund verstanden werden, auch nicht als ein absolutes Subjekt, sondern eigentlich nur als ein Gott, der Beziehung und in Beziehung Leben ist, gelebte Liebe. Und das ist der Grund, warum Gott - ausgehend von Jesus als dem Mensch gewordenen Gottessohn - im Christentum als der drei-eine Gott verstanden wird: Gott-Vater und Gott-Sohn verbunden in gegenseitiger Liebe, die Gottes Heiliger Geist ist.

Nirgendwo wird dieser Gott in seiner Liebe deutlicher erkennbar als am Kreuz, in der Hingabe Jesu bis dorthin, dass er sich nicht nur von den Menschen, sondern auch von Gott verlassen glaubt: „Mein Gott, mein Gott, warum hast Du mich verlassen?!“( Mk 15,34). Dem entsprechend bringt Chiara Lubich ihre Lebensentscheidung in einem frühen Text so zum Ausdruck: „Ich habe nur einen Bräutigam auf der Erde: den gekreuzigten und verlassenen Jesus; ich habe keinen anderen Gott außer ihm. ${ }^{\text {“6 }}$

\section{Gelebter, nicht nur gedachter Glaube}

Wenn Gott ein drei-einer Gott, wenn er in sich gelebte Liebe, Beziehung ist, dann liegt es nahe bzw. entspricht es dem, dass eine Spiritualität, die diesen Gott zu ihrer Mitte hat, sich - wie bereits gezeigt - ebenfalls der Liebe und Beziehungen verpflichtet weiß. Das so entstandene Leben der gegenseitigen Liebe und Einheit in Gott auf der Grundlage eines Paktes, die Eucharistie und das Leben des Wortes führen im Jahr 1949 zu Erfahrungen, die offensichtlich nicht anders denn als Paradies bezeichnet werden konnten - eine überwältigende Bestätigung dafür, dass die in Gott gelebte Einheit selbst göttlich ist. Es ist die Erfahrung, dass Menschen, die diese Einheit leben, ihren Ort in Gott, im „Schoß des Vaters“ finden. Das ist in erster Linie Gnade, also Geschenk; dann aber auch Auftrag: Was immer in der Gesellschaft gelebt wird, soll in dieses neue, von Gott geprägte Leben einbezogen werden.

\section{Wenn Beziehung und Bewegung in die Mitte des} Interesses rücken. Oder: Trinitarische Ontologie

Die eben skizzierte Spiritualität der Einheit hat auf Klaus Hemmerle erheblichen und besonderen Einfluss ausgeübt. Und das gilt, obwohl er in seiner

\footnotetext{
6 Ch. Lubich, Bis wir alle eins sein werden, München $1983^{12}$, S. 108.
} 
wissenschaftlichen Laufbahn verschiedene philosophisch und theologisch Autoren und Denkstile kennengelernt hat und von ihnen geprägt worden ist. $\mathrm{Zu}$ erinnern ist an seinen Lehrer, den phänomenologisch geprägten Bernhard Welte; Franz vom Baader und Bonaventura, denen er große wissenschaftliche Arbeiten gewidmet hat; und nicht zuletzt Heinrich Rombach mit seiner Strukturontologie, ein Werk, das Hemmerle ausführliche rezensiert hat.

\section{Eine Schule des Denkens}

Trotz dieser vielfältigen Einflüsse hat für Hemmerle die Spiritualität der Einheit besondere Bedeutung gewonnen. Noch in seinem letzten Beitrag, den er knapp vor seinem Sterben verfasst hat, hat Klaus Hemmerle darauf hingewiesen, dass die geistlichen Intuitionen des Charismas der Einheit für ihn nicht nur geistlich-existenziell, sondern auch als Theologe prägend geworden sind. Er schreibt: „Chiara Lubich hat uns in eine Schule des Lebens genommen, aber diese Lebensschule ist zugleich auch eine Schule für die Theologie. Das Ergebnis ist nicht eine Verbesserung der Theologie, sondern gelebte Theologie aus dem Ursprung der Offenbarung. " testen Ausdruck hat das Denken, das er im Charisma der Einheit entdeckt hat, in seinen 1976 erschienenen Thesen zu einer trinitarischen Ontologie gefunden. ${ }^{8}$

\section{Ausgehend vom „dreifaltigen Ereignis“}

Die Grundidee dieser Thesen kann so umrissen werden: Es geht darum, die Wirklichkeit im Licht des Glaubens an den drei-einen Gott zu verstehen: Mit anderen Worten: Das, was die Wirklichkeit zur Wirklichkeit macht, also ihre ontologische Tiefenstruktur muss als Wirklichkeit in Beziehung gedacht werden. Grund dafür ist die Tatsache, dass auch Gott Wirklichkeit in Beziehung ist. ${ }^{9}$

Dem entsprechend geht Klaus Hemmerle bei seinem Entwurf einer trinitarischen Ontologie von der christlichen Gotteserfahrung aus. Diese Erfahrung versteht und charakterisiert er als „das dreifaltige Ereignis ${ }^{{ }^{10}}$ - das sei das unterscheidend Christliche. ${ }^{11}$ Dieses Ereignis ist für Hemmerle in Jesus

K. Hemmerle, Unser Lebensraum - der dreieine Gott, „Das Prisma“ 1 (1994), S. 23.

8 Daneben ist als weitere Quelle für Hemmeles Thesen zu einer trinitarischen Ontologie vor allem die Strukturontologie von Heinrich Rombach zu nennen.

9 Diese Sicht ist seit Augustinus mit seinem epochalen Werke De Trinitate und seiner Rede von den „substanziellen Relationen“ (also Beziehungen, die das Wesen Gottes ausmachen) in der westlichen Theologie unbestritten.

${ }^{10}$ Hemmerle: These 15.

${ }^{11}$ Vgl. Hemmerle, Thesen 18. 
Christus gegeben. In ihm kommt „Gott ganz hinein in die Geschichte - und bleibt doch über ihr. ${ }^{“ 12}$ So verwandelt sich das Bild Gottes. Gott ist Beziehung zwischen „Gott über uns" und „Gott unter uns“, die geeint sind im Heiligen Geist der Liebe. Die Rede von der Trinität ist deshalb für Hemmerle nicht vermessene Spekulation, sondern sie bringt zum Ausdruck, „wie dem Menschen Gott und wie der Mensch sich selbst neu geschenkt ist, indem er Jesus Christus glaubt. ${ }^{{ }^{113}}$ Das Stichwort dieses neuen Verständnisses von Gott und Mensch lautet „Liebe“, oder anders: „Sich-geben“. ${ }^{14}$

Diese Zusammenhänge bilden für Hemmerle den Ausgangspunkt und die spezifische Prägung seiner trinitarischen Ontologie. Gemessen an bisherigen Entwürfen der Ontologie markiert sie für Hemmerle eine revolutionäre Wende.

\section{Beziehung und Bewegung als Schlüsselbegriffe}

So heißt es bei Klaus Hemmerle: „Die revolutionierende Kraft des schlichten Wortes, daß allein die Liebe bleibt, ist kaum zu überschätzen. Denn wenn das Bleibende die Liebe ist, dann ist die Verlagerung des Schwerpunktes aus dem Selbst ins Andere, dann ist die (nicht mehr aristotelisch verstandene) Bewegung, dann ist die (ebenfalls nicht mehr als Kategorie, gar als seinsschwächstes Akzidens verstandene) relatio in die Mitte gerückt. Aber Beziehung, Bewegung werden nicht als ein neues Prinzip statuiert, aus dem sich alles doch wiederum in einsamer Deduktion ableiten ließe. Nur eines bleibt: das Mittun jener Bewegung, welche die agape selbst ist. Diese Bewegung ist der Rhythmus des Seins; es ist der Rhythmus des Gebens, das sich selber gibt." ${ }^{15}$

Mit diesem Absatz rückt Hemmerle die entscheidende Weichenstellung in den Blick, die er mit einer trinitarischen Ontologie vornimmt. Zwei Aspekte sollen herausgehoben werden: (1.) Eine Ontologie ist immer an der Frage interessiert, was die Wirklichkeit zur Wirklichkeit macht. Damit ist ein Interesse am Bleibenden gegeben. Wenn aber - mit Hemmerles Worten - „allein die Liebe bleibt“, dann wird das Bleibende nicht in einem statisch verstandenen Wesen festgemacht, sondern in einer Dynamik. Wenn das Wesen der Wirklichkeit Liebe (agape) ist, dann sind „Beziehung“ und „Bewegung“ das Bleibende.

Aber (2.) Beziehung und Bewegung sind für Hemmerle nicht nur Gegenstand der Erkenntnis und der Erörterung bzw. ein neues Prinzip, sondern sie

\footnotetext{
${ }^{12}$ Hemmerle: These 15.

${ }_{13}$ Hemmerle: These 15.

14 Vgl. Hemmerle: These 18.

${ }^{15}$ Hemmerle: These 18.
} 
bestimmen auch den Vorgang der Erkenntnis. Erkenntnis geschieht dadurch, dass Bewegung und Beziehung als „Rhythmus des Seins“ mitvollzogen werden, im „Rhythmus des Gebens, das sich selber gibt“ - wie Hemmerle schreibt. Ein Erkennen, das die Wirklichkeit so wahrnimmt wie sie ist, gelingt nur wenn man sich auf den Rhythmus einlässt, der die Wirklichkeit bestimmt. Mit anderen Worten: In Hemmerles Ontologie geht es nicht nur um neue Inhalte, sondern - grundlegender - um ein neues Denken.

\section{Verdeutlicht am Beispiel des Wortes}

Das heißt: Was die Wirklichkeit zur Wirklichkeit macht, das ist für Hemmerle nicht ein statisches Wesen, sondern die Wirklichkeit ist Ereignis in Beziehungen und Bewegung. Hemmerle verdeutlicht diese Einsicht am Beispiel des Wortes. ${ }^{16}$ Eine Möglichkeit zu verstehen, was das Wort ist, könnte darin bestehen, dass man eine Umschreibung vornimmt - indem man etwa sagt: "Ein Wort ist ein sprachliches Element mit einer bestimmten Bedeutung“. Hemmerle wählt einen anderen Weg. Ihm geht es nicht um die Frage, was ein Wort ist, sondern wie es geht, wie in einem bestimmten Vollzug das Wort zum Wort wird. Und das geschieht - so Hemmerle - in Beziehungen und Bewegung: Das Wort wird bestimmt durch den, er es spricht. Es wird aber auch durch den bestimmt, zu dem es gesprochen wird. Und schließlich wird es auch bestimmt durch die Sprache, der es angehört.

Damit wird deutlich: Hemmerle gibt der konkreten Wirklichkeit den Vorrang vor einer allgemein verstandenen, aber abstrakten Wesensbestimmung der Wirklichkeit. Damit weist er sich als Anwalt des phänomenologischen Denkens aus, das sich nicht primär auf der Ebene abstrakter Wesensbestimmungen abspielt, sondern sich der Wirklichkeit aussetzt. „Zu den Sachen selbst“ ist das Motto, dass der Philosoph Edmund Husserl dieser von ihm begründeten Denkschule mitgegeben hat.

\section{Aufmerksamkeit für das Dazwischen. Oder: einige Hinweise zur Anwendung}

Klaus Hemmerles trinitarische Ontologie ist also - so wie auch jede andere Ontologie, die ausformuliert vorgestellt wird - eine Einladung, die Wirklichkeit auf eine bestimmte Weise wahrzunehmen und ihren Einsichten entsprechend zu leben.

\footnotetext{
16 Vgl. Hemmerle: These 21.
} 
Ein neues Denken, Sehen, Erkennen

„Auf eine bestimmte Weise wahrnehmen“ - genau darum geht es. Einerseits soll durch Hemmerles trinitarische Ontologie etwas Neues sichtbar und auch denkbar werden. Andererseits leitet sie nicht nur an, etwas Neues zu sehen, sondern sie leitet auch zu einem neuen Sehen an. Oder wie Hemmerle es im oben zitierten Absatz formuliert: Es geht im Sehen, in der Erkenntnis um das „Mittun jener Bewegung, die die Agape ist “ ${ }^{17}$ Es geht um ein Denken, das tatsächlich oder wenigstens der Absicht nach Denken in Beziehung und Bewegung ist.

\section{Das Dazwischen rückt ins Blickfeld}

Hemmerle nimmt - wie gezeigt - als Ausgangspunkt seiner trinitarischen Ontologie das „dreifaltige Ereignis“. Ereignis - diese Formulierung ist kein Zufall: Gott wird nicht als eine statische Wesenheit ins Spiel gebracht, sondern als Geschehen. Etwas pointiert (und vielleicht auch etwas missverständlich) formuliert: Wenn Gott als der drei-eine Gott gedacht wird, dann ist Gott nicht der Vater oder der Sohn oder der Heilige Geist, sondern ein Gott in lebendigen Beziehungen - zwischen Vater, Sohn und Heiliger Geist. Gott wird als ein Gott gedacht, für den die Beziehungen zwischen den Personen und damit das Dazwischen zu seinem Wesen gehört. Noch einmal etwas pointiert gesagt: Das Dazwischen ist der Ort, an dem Gott zum Ereignis wird.

Und die Welt im Licht dieses Gottes bedacht, wird damit ebenfalls zu einer Wirklichkeit, für die Beziehung, Bewegung und damit das Dazwischen konstitutiv werden und auf neues Interesse stoßen. In der von Hemmerle konzipierten trinitarischen und damit relationalen Ontologie ist diese Sicht nicht nur eine Option unter mehreren, sondern die Option, die es möglich macht, die Wirklichkeit angemessen zu verstehen und sich angemessen in der Wirklichkeit zu bewegen. In einem relationalen Denken wird das Dazwischen, das im ersten Augenblick als eine zu vernachlässigende Größe, als Niemandsland erscheinen mag, als der eigentlich fruchtbare Ort des Erkennens und des Lebens. Das soll abschließend an zwei Beispielen sichtbar gemacht werden.

\section{Denken im Dazwischen}

Lesen ist Abenteuer im Kopf - so hat es in einem Werbespruch geheißen. Das gilt natürlich auch für das Denken. Zutreffend signalisiert der Begriff Abenteuer, dass Denken nicht nur eine Chance ist, sondern oft genug auch Kühnheit. Das haben nicht zuletzt die Theologen mit ihren auf Gott zielenden

\footnotetext{
${ }_{17}$ Hemmerle: These 18.
} 
Denkbewegungen immer schon gewusst. Und ganz allgemein gilt: Wer denkt, begibt sich auf ausgesetzte Wege - Irrwege und Abstürze nicht ausgeschlossen.

Kein Wunder, dass man nach Möglichkeiten gesucht hat, Denkwege durch verlässliche und bewährte Methoden (meta-hodos) abzusichern. Das macht natürlich Sinn. Und es kann gegen bewusste Irreführungen durch Sophisten schützen. Ursprung und Entwicklung der Logik und ihrer „Zwillingsschwester", der Rhetorik illustrieren das.

Aber es ändert nichts daran, dass Denken immer wieder neu zum Abenteuer wird. Denken, das ist nicht nur Deduktion, also regelkonforme Ableitung einer neuen Aussage aus zwei bereits als wahr erkannten Aussagen. Ein solches Schlussverfahren bringt im Grunde nichts Neues. Fruchtbares Denken ist ein zuweilen und immer wieder ein riskantes Spiel, dessen Ausgang offen und ungewiss ist. Und gerade auf diese Weise kann es Neues entdecken, an die Grenze des Erkennens gehen und darüber hinaus.

Solches Denken ist Denken im Dazwischen. Ein Beispiel aus der reichen Geschichte der Philosophie und Theologe ist das analoge Denken, also das Regelwerk für das Erkennen Gott und das Sprechen über ihn. Im Anschluss an die Predigt Jesus sprechen wir Gott als Vater an. Und wir wissen natürlich, dass er nicht Vater im Sinn eines irdischen Vater ist, sondern auf seine, also göttliche Weise. Im Dazwischen von Ja (Gott ist Vater), Nein (Gott ist nicht im irdischen Sinn Vater) und Steigerung (Gott ist auf göttliche Weise Vater) nähern wir uns der Erkenntnis der Wirklichkeit Gottes.

Aber man kann vermutlich ohne viel Aufwand zeigen, dass es viele Wirklichkeiten gibt, nicht zuletzt Menschen, die nicht durch eine Aussage allein erfasst werden können, sondern nur im Spannungsfeld vieler, komplementärer und vielleicht sogar widersprüchlicher Aussagen angemessen erkannt werden können und müssen. Das kann verunsichern und ängstigen: Verlieren wir da nicht alle Sicherheiten? Wird nicht alles relativ und gleichgültig? Hier bleiben in diesem Beitrag sicher einige wichtige Fragen offen, die noch einer Klärung bedürfen. Das hat nicht zuletzt auch Klaus Hemmerle in seinen Thesen gesehen und angesprochen. ${ }^{18}$

\section{Im Dazwischen unter Menschen}

Der Mensch ist ein zoon politicon, ein soziales und politisches Wesen, also ein Wesen in Gemeinschaft. Gemeinschaft kann man natürlich als Addition von Individuen sehen, aber man weiß sofort, dass die Aufzählung ihrer Mitglieder

18 Vgl. Hemmerle: These 23. 
keine angemessen Darstellung einer Gemeinschaft ist. Dazu gehört mehr. Gemeinschaften sind Geflechte von Beziehungen, von vielfältigen geistigen, emotionalen und handelnden Interaktionen, Gemeinschaften sind gelebte produktive Spannungsfelder. Gemeinschaften sind vielgestaltige Beziehungen und Bewegungen.

Und so ist noch einmal das Dazwischen ins Blickfeld gerückt. Eine relationale Ontologie setzt dabei bei Einsichten an, die durchaus bereits bekannt waren und sind. Aber sie ist zugleich eine Einladung, das Dazwischen in seinen vielen Facetten besonders zu beachten und zu werten.

Dabei geht es nicht um ein Schlaraffenland. Beziehungen und das durch sie eröffnete Dazwischen können Orte großartiger Erfahrungen sein, aber auch Konfliktzonen. Das Dazwischen von Beziehungen kann Niemandsland sein, das man hinter sich bringen will oder für das man als Schutzzone dankbar ist, aber auch eine Möglichkeit, in eine neue Welt aufzubrechen. Das Dazwischen kann eine Verheißung sein, aber auch verunsichern und erschrecken. Denn in Beziehungen setzt man sich dem Anderen aus.

Deshalb wird man immer versuchen, das in-Beziehungen-treten auf verschiedene Weise abzusichern. Das aber bringt die Gefahr mit sich, dass man den Anderen nicht als Anderen gelten lässt. Wenn man dagegen den Anderen als Anderen ernst nehmen will, dann ist das Dazwischen nicht nur der Ort für eine Chance, sondern auch der Ort eines Risikos. Und es verlangt den manchmal mühevollen und verunsichernden Weg des Zuhörens, eines Dialogs, der nicht vereinnahmt, sondern den Anderen als Anderen gelten lässt und sogar damit rechnet, dass er gerade als der Andere wertvoll ist - nicht zuletzt für mich.

Und damit ist das Ende dieser Überlegungen erreicht. Wenn man dem Gesagten treu bleiben will, dann kann das Ende nur darin bestehen, dass wir im erreichten Zwischenraum bleiben. Vielleicht machen wir im anschließenden Gespräch die Erfahrung, dass er nicht Niemandsland ist, sondern ein vielleicht mühsamer, aber fruchtbarer Raum der Erkenntnis und des Lebens. 


\section{Abstract}

\section{The God of the "in-Between".}

Based on the theses of Klaus Hemmerle's trinitarian ontology and Chiara Lubich's spirituality, the article shows the practical consequences of relational ontology. Its content includes the following issues: basic assumptions in everyday life and ontology; the charism of unity and its transformation into trinitarian ontology; the "in-between" as essential for the understanding of God and reality.

\section{KEYWORDS}

Ontology, trinitarian ontology, relational ontology, Trinity, charisma of unity, triune event, Lubich Chiara, Hemmerle Klaus

\section{ABSTRAKT}

\section{Bóg tego, co „pomiędzy”}

W oparciu o tezy ontologii trynitarnej Klausa Hemmerlego i duchowość Chiary Lubich artykuł pokazuje praktyczne konsekwencje ontologii relacyjnej. Jego treścią są następujące zagadnienia: istota ontologii i jej podstawowe założenia, „charyzmat jedności” i jego przekształcenie w ontologię trynitarną, doniosłość „tego, co pomiędzy" dla adekwatnego rozumienia Boga i rzeczywistości.

\section{SŁOWA KLUCZOWE}

ontologia, ontologia trynitarna, ontologia relacyjna, charyzmat jedności, Trójca Święta, wydarzenie troiste, Lubich Chiara, Hemmerle Klaus

\section{LITERATUR}

Augustinus, De Trinitate, in: S. Aurelii Augustini Opera omnia, t. 8, ed. J.-P. Migne, Parisiis 1841, Sp. 819-1098 (Patrologiae Cursus Completus. Series Latina, 42).

Hemmele K., Thesen zu einer trinitarischen Ontologie, Einsiedeln 1976.

Hemmerle K., Lontologia del "Paradiso" '49, „Sophia: Ricerche su i fondamenti e la correlazione dei saperi" 6 (2014) n ${ }^{\circ} 2$, S. 127-137.

Hemmerle K., Unser Lebensraum - der dreieine Gott, „Das Prisma“ 1 (1994), S. 17-23. Körner B., Il Dio dell'in-mezzo, „Sophia: Ricerche su i fondamenti e la correlazione dei saperi“ 8 (2016) $n^{\circ} 2$, S. 61-71.

Lubich Ch., Bis wir alle eins sein werden, München $1983^{12}$. 
Lubich Ch., Die Welt wird eins. Franca Zambonini im Gespräch mit der Gründerin der Fokolar-Bewegung, München 1991.

Rombach H., Strukturontologie. Eine Phänomenologie der Freiheit, Freiburg-München $1988^{2}$.

Strawson P. F., Einzelding und logisches Subjekt, Stuttgart 1972. 